\title{
Noise and Synchronization Analysis of the Cold-Receptor Neuronal Network Model
}

\author{
Ying Du, ${ }^{1}$ Rubin Wang, ${ }^{1}$ and Jingyi $Q \mathbf{u}^{2}$ \\ ${ }^{1}$ Institute for Cognitive Neurodynamics, School of Science, East China University of Science and Technology, Shanghai 200237, China \\ ${ }^{2}$ School of Information Science and Technology, Civil Aviation University of China, Tianjin 300300, China
}

Correspondence should be addressed to Ying Du; du.ying.buaa@gmail.com

Received 27 January 2014; Accepted 25 May 2014; Published 15 June 2014

Academic Editor: Wenwu Yu

Copyright (C) 2014 Ying Du et al. This is an open access article distributed under the Creative Commons Attribution License, which permits unrestricted use, distribution, and reproduction in any medium, provided the original work is properly cited.

\begin{abstract}
This paper analyzes the dynamics of the cold receptor neural network model. First, it examines noise effects on neuronal stimulus in the model. From ISI plots, it is shown that there are considerable differences between purely deterministic simulations and noisy ones. The ISI-distance is used to measure the noise effects on spike trains quantitatively. It is found that spike trains observed in neural models can be more strongly affected by noise for different temperatures in some aspects; meanwhile, spike train has greater variability with the noise intensity increasing. The synchronization of neuronal network with different connectivity patterns is also studied. It is shown that chaotic and high period patterns are more difficult to get complete synchronization than the situation in single spike and low period patterns. The neuronal network will exhibit various patterns of firing synchronization by varying some key parameters such as the coupling strength. Different types of firing synchronization are diagnosed by a correlation coefficient and the ISI-distance method. The simulations show that the synchronization status of neurons is related to the network connectivity patterns.
\end{abstract}

\section{Introduction}

It is expected that the processed neural information can be encoded in the structure of inter-spike-interval (ISI) series; that is, the neural firing activities can be represented by the patterns of neural spike trains [1-4].

Since the introduction of the concept of stochastic resonance [5], a positive role of noise has become an important issue in physics and related neural systems. Nonlinear systems can change their dynamics with the addition of noise $[1,6]$. Most neural models consist of differential equations that can reproduce observed neuronal behavior. When these models are stimulated by noise, a variety of phenomena including stochastic resonance and coherence resonance will be observed [7-10]. Besides the deterministic state that triggers the membrane potential of neurons, firing spike train is also affected by noise [11]. For example, for subthreshold oscillations near the threshold of spike generation, it is dependent on noise whether a spike is triggered or not. Spike activity with skipping can be fully understood only when noise is considered. Transitions of spike train patterns are also affected by noise [12]. With the addition of noise, it was observed there were a variety of spike train patterns in temperature sensitive skin receptors for information encoding $[13,14]$. The corresponding data analyses suggest that noise can extend the dynamical behavior of the system $[15,16]$. Similar results can also be observed in experiment [17-20], which indicate that the nervous system may take advantage of the benefits of noises, including internal thermal fluctuations and external noisy inputs.

Except the contribution of a single neuron on information transmitting, synchronization of a set of interacting units has been intensively studied in the natural world [21, 22]. The synchronization of neuronal signals has been proposed as one of the mechanisms to encode the signal $[23,24]$. It is suggested that theoretical studies of synchronized behaviors in neuronal system play an important role in information processing of olfactory systems. Synchronous behavior of two electrically coupled neurons was studied by Postnova et al. [25]. Asynchronous and other synchronous states were investigated too. The results showed that the coupling strength could have important role on the synchronous states. Synchronization of fast-spiking neurons connected by electrical synapses was investigated by Nomura and his team [26]. 
Many different measures have been introduced to characterize effects of noise and the synchronization transaction of neurons [26-29]. There exist different connectivity patterns including ring-like neuronal network and grid-like neuronal network. The former only considers the coupling of nearest neighbor neurons, while the latter includes all the nearest neighbor connected couplings. Synchronization studies between two coupled neurons and multiple neural networks are studied [30-34].

Except the traditional methods, in this paper, ISI distance, a new method recently introduced by Kreuz et al. [35, 36], is used to characterize effects of noise in a Hodgkin-Huxley type model of temperature encoding and synchronization. This is a simple approach that extracts information from the interspike intervals by evaluating the ratio of the instantaneous firing rates, which is complementary to the spike-based approaches. With the addition of noise, the neural model can generate a variety of new different patterns compared to deterministic situation; synchronization status of neural network with different connection types is also investigated, and the simulations show that the synchronization status is much related to the network connectivity patterns.

The paper is organized as follows: the model of neural system is introduced in Section 2. Noise effects on neural system are characterized in Section 3, including bifurcation plots and ISI distance about the noise effects. Section 4 presents the synchronization results of two-coupled neurons and neuronal networks with different connection patterns, showing the different states of synchronization by varying control parameters, the coupling strength. Finally, a brief conclusion is given in Section 5.

\section{The Neural Model}

A simplified Hodgkin-Huxley model [16] is used to simulate the spike train activity of cold receptor response to temperature changes in the skin. This model consists of two sets of ionic conductance, each related to de- and repolarizing currents:

$$
\begin{aligned}
C_{M} \frac{d V}{d t} & =f(V) \equiv-g_{l}\left(V-V_{l}\right)-\alpha\left(I_{d}+I_{r}\right)-\beta\left(I_{s d}+I_{s r}\right) \\
I_{i} & =\rho g_{i} a_{i}\left(V-V_{i}\right), \quad(i=d, r, s d, s r) \\
a_{i \infty} & =\frac{1}{\left(1+\exp \left(-s_{i}\left(V-V_{0 i}\right)\right)\right)} \\
\frac{d a_{i}}{d t} & =\frac{\phi\left(a_{i \infty}-a_{i}\right)}{\tau_{i}} \\
a_{d} & =a_{d \infty}
\end{aligned}
$$

where $V$ is the membrane potential, $C_{M}$ is the membrane capacitance, and the leakage currents are given by $g_{l}(V-$ $\left.V_{l}\right) . I_{d}$ and $I_{r}$ are the fast depolarizing current and the repolarizing one for spike generation, and two slow currents for subthreshold oscillations are given by $I_{s d}$ and $I_{s r} . \alpha$ and $\beta$ are parameters used to alter the spike currents, especially for $a_{s r}$

$$
\frac{d a_{s r}}{d t}=\frac{\phi\left(-\eta I_{s d}-k a_{s r}\right)}{\tau_{s r}} .
$$

$V_{i}$ are the equilibrium potentials, $g_{i}$ are the conductance, and $V_{0 i}$ and $s_{i}$ are the half-activation potentials and the slopes of sigmoidal steady-state activation curves. The constant $\eta$ is the coupling contrast, while $k$ is the relaxation factor. Temperature dependencies are introduced by the parameters $\rho$ and $\phi$, respectively,

$$
\rho=1.3^{\left(T-T_{0} / 10\right)}, \quad \phi=3.0^{\left(T-T_{0}\right) / 10},
$$

where $T$ is the temperature and $T_{0}$ is the reference temperature.

\section{Noise Effects on the Neural System}

In order to study the stochastic disturbances on the model, noise is implemented to the membrane potential Equation (1) in each time step $\Delta t$ according to the Box-Mueller algorithm [37]:

$$
\begin{gathered}
V_{t+\Delta t}=V_{t}+\left(\frac{f(V)}{C_{M}}\right) \Delta t+g_{w}, \\
g_{w}=(-4 d \ln (a) \Delta t)^{1 / 2} \cos (2 \pi b),
\end{gathered}
$$

where $a, b$ are random numbers between 0 and $1, d$ is the noise intensity, and the noise $g_{w}$ is determined by its Gaussian statistical features.

In the deterministic situation without noise, the neural model described above exhibits different types of spiking patterns when the parameter of temperature $T$ is varying. At low temperature, spike pattern is period single firing, and then for slightly higher temperature the firing pattern changes into chaos firing. When $T$ is increasing to $15^{\circ} \mathrm{C}$, spike train patterns become bursts, and the number of spikes per burst will be reduced if the temperature is further increased (up to $T=20^{\circ} \mathrm{C}$ ), for high temperatures again regular single-spike activity occurs $\left(T=35^{\circ} \mathrm{C}\right)$, but with higher spiking frequency. These varying processes of spike train pattern can be seen from the ISI plot shown in Figure 1(a), in which successive interspike intervals are plotted versus time as a function of temperature scaling. Starting form high temperatures, the behavior of the spike firing patterns changes from subthreshold oscillations to high-frequency regular single spike. At lower temperatures, there are several abrupt transitions from period one to period four with the number of spikes per burst increases. At temperature around $10^{\circ} \mathrm{C}$, increasing the number of bifurcations occurs followed by chaotic patterns. At still lower temperature, regular singlespike activity with lower frequency appears again.

In the noisy simulation, the neural model exhibits temperature dependencies that well follow the main characteristics of the experimental data. When especially $d$ is taken as 0.5 , some fluctuations of spike timing are visible as shown in Figure 1(b). At $T=35^{\circ} \mathrm{C}$, instead of regular singlespike generation, two spikes per oscillation cycle appear. 


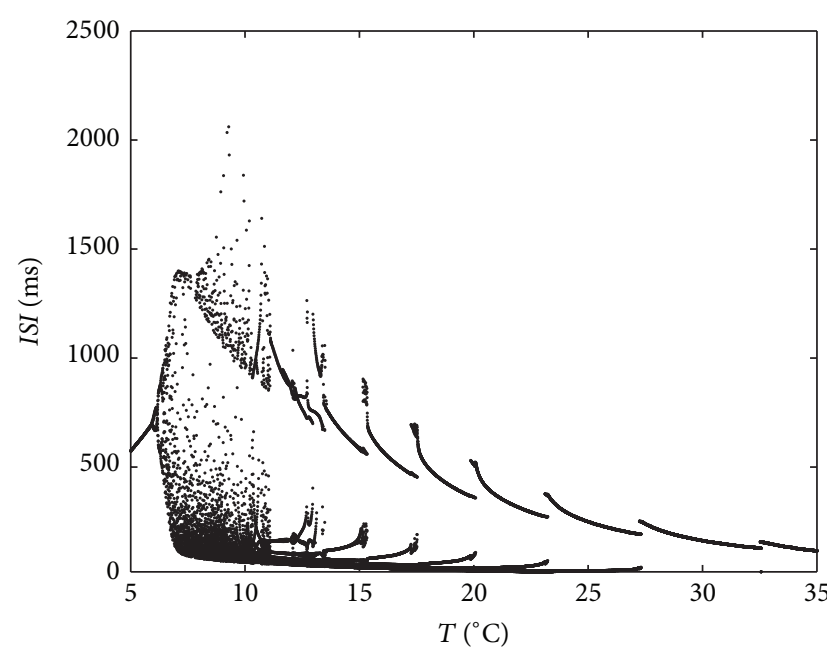

(a)

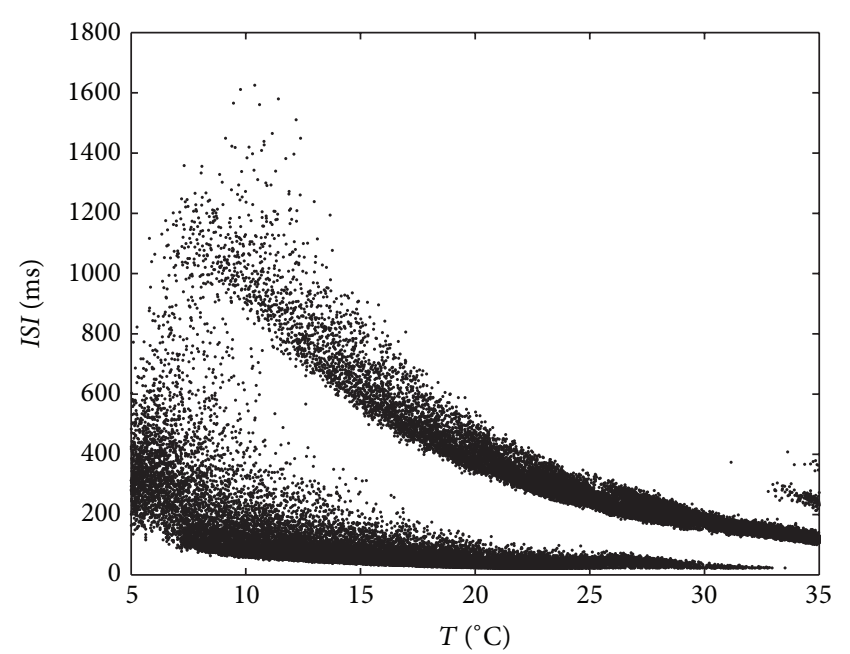

(b)

FIGURE 1: ISI plots with respect to variation of the temperature $T$ in the deterministic situation and the noise situation with $d=0.5$.

At mid temperatures, the abrupt transitions of deterministic simulation are completely smoothed out by noise, which can be seen in the interval plot as shown in Figure 1(b). The qualitative differences occur in the lower and higher temperature situations, in which skipping in the noisy simulations appears and the transitions to chaotic interval sequences in deterministic simulations disappear.

ISI distance is an useful method to qualitative the effects of noise on the neuron system [35]. This approach is based on the time intervals between successive spikes as the basic element for measuring the difference of the two spike trains qualitatively. Due to the self-adaptation of this method, it can naturally describe the characterization of neuronal spike trains. The ISI distance between two spike trains $x(t)$ and $y(t)$ is introduced briefly by the following procedure. First, define the interspike interval of the spike train $x(t)$ at time $t$ by

$$
\begin{array}{r}
x_{i s i}(t)=\min \left(t_{i}^{x} \mid t_{i}^{x}>t\right)-\max \left(t_{i} \mid t_{i}^{x}<t\right), \\
i=1, \ldots, M, \quad t_{1}^{x}<t<t_{M}^{x},
\end{array}
$$

where $t_{i}^{x}$ is the time of the $i$ th spike of the spike train $x(t)$. In the same manner, define the function of $i$ th spike of $y(t)$, and then the normalized ratio $I(t)$ is taken as follows:

$$
I(t)=\frac{x_{i s i}(t)-y_{i s i}(t)}{\max \left(x_{i s i}(t), y_{i s i}(t)\right)} .
$$

Finally, the ISI distance $D_{I}$ is computed through integrating over time as follows:

$$
D_{I}=\int_{0}^{t_{M}} d t|I(t)|
$$

Here, this measure is used to qualitatively characterize the difference between deterministic and noisy spike trains of the cold receptor. Figure 2 shows the change of ISI distances as a continuous function of temperature. As shown in the picture, at each fixed temperature, the ISI distance increases

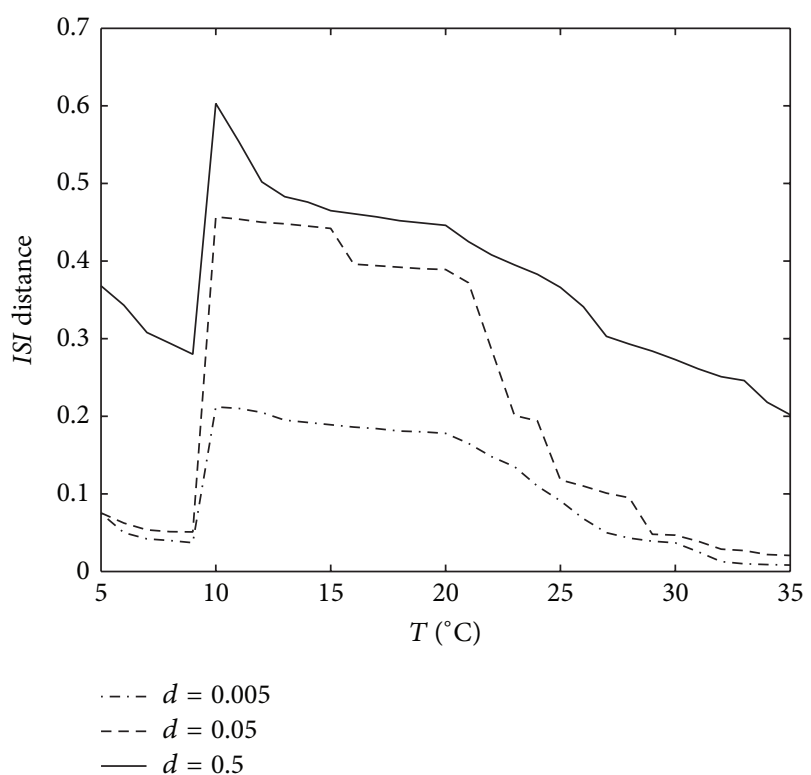

FIgURE 2: The change of ISI distance as a function of temperature T at different noise densities. Solid line: $d=0.5$; dashed line: $d=0.05$; dot-dashed line: $d=0.5$.

with the noise intensity becoming larger. For example, at $T=10^{\circ} \mathrm{C}$, when $d$ is taken as $0.005,0.05$, and 0.5 , the ISI distance is $0.212,0.457$, and 0.603 , respectively, which means that the higher noise intensity causes stronger fluctuations of the times in the spike train.

From the study of noise effect in the neural system, we can obtain that, with the addition of noise, the neural model can generate a variety of different spike train patterns and allow continuous transitions from bursting to subthreshold oscillations. Thus, the system with noise may be more close to actual dynamics underlying the experimental data. 


\section{Synchronization Study in Neural Network System}

In the process of information transmitting and code, synchronization of firing plays an important role. Hence in this section, synchronization of two coupled neurons and network is studied.

Dynamics of multiple coupled neurons in this paper are controlled as shown by the following differential equations:

$$
\begin{aligned}
C_{M} \frac{d V_{i}}{d t}= & f(V) \\
\equiv & -g_{l}\left(V_{i}-V_{l}\right)-\alpha\left(I_{d}+I_{r}\right)-\beta\left(I_{s d}+I_{s r}\right) \\
& +I_{\text {couple }}(i), \\
I_{\text {couple }}(i)= & \sum_{j} g_{c}\left(V_{i}-V_{j}\right),
\end{aligned}
$$

where $g_{c}$ is coupling strength and $V_{i}$ and $V_{j}$ are voltages between adjacent neuron $i$ and $j$. For two bidirectional coupled neurons (Figure $3(\mathrm{a})$ ) $, i, j=1,2$; for the ring-like network with $N$ neurons (Figure 3(b)), $I_{\text {couple }}(i)$ is the sum of the adjacent neurons including previous and next ones:

$$
I_{\text {couple }}(i)=g_{c}\left(V_{i}-V_{i-1}\right)+g_{c}\left(V_{i}-V_{i+1}\right) \quad i=1,2, \ldots, N .
$$

For the grid-like network (Figure 3(c)), the $I_{\text {couple }}(i, j)$ of a neuron at position $(i, j)$ is the sum of the input currents from the nearest neighbor neurons:

$$
I_{\text {couple }}(i, j)=\sum g_{c}\left(V_{i, j}-V_{i+n, j+m}\right) \quad i, j=1,2, \ldots, N .
$$

4.1. Synchronization of Two Coupled Neurons. First, synchronization status of two identical coupled neurons is considered. The ISI distance method introduced above is used to estimate the degree of synchrony between two spike trains. As introduced in Section 3, the quantity of ISI ratio $I_{1,2}(t)$ will be zero if two spike trains are completely alike, or it will approach -1 and 1 , respectively, if two spike trains differ greatly. So the more synchronous the coupled neurons are, the less the ISI distance $D_{I}$ is, and $D_{I}$ is equal to 0 when the complete synchronization state of the coupled neurons is achieved.

For two identical coupled neurons as Figure 3(a), synchronization of the two-coupled neurons both at regular single spiking is investigated and the numerical result is shown in Figure 4 by varying the coupling strengths. In the figure, the change of the ISI distance ((10) and (11)) as a function of the increasing coupling strength is shown. It increases to its maximal value when $g_{c}=0.013 \mathrm{~ms} / \mathrm{cm}^{2}$, before that, the ISI distance is increasing due to chaotic behavior of the neuron. Then it decreases gradually to 0 in the range of $0.013<$ $g_{c}<0.03 \mathrm{~ms} / \mathrm{cm}^{2}$. Finally, it remains as 0 when $g_{c}$ is larger. The ISI distance here shows that the synchronous degree of two neurons first decreases and then increases to complete synchronization when $g_{c}$ is large enough. Similar results can be found in other identical coupled neurons with different firing patterns (bursting, chaos firing, etc.).
Another method to characterize the synchrony degree between two spike trains is the correlation coefficient (CC), and the CC is calculated as follows:

$$
\mathrm{CC}=\frac{\sum_{i-1}^{n}\left|\left(V_{i}^{1}-\left\langle V_{i}^{1}\right\rangle\right)\right|\left|\left(V_{i}^{2}-\left\langle V_{i}^{2}\right\rangle\right)\right|}{\sqrt{\sum_{i-1}^{n}\left(V_{i}^{1}-\left\langle V_{i}^{1}\right\rangle\right)^{2}\left(V_{i}^{2}-\left\langle V_{i}^{2}\right\rangle\right)^{2}}},
$$

where $V_{i}^{1}$ (or $V_{i}^{2}$ ) is the sampling of the membrane potential $V^{1}(t)$ (or $\left.V^{2}(t)\right)$. $\langle\cdot\rangle$ calculates the average over the number of the sampling. So the larger the correlation coefficient is, the more synchronous the two coupled neurons are, and the complete synchronization will be achieved when correlation coefficient is equal to 1 .

As illustrated in Figure 5, the correlation coefficient value (15) increases gradually from 0 to 1 when $g_{c}$ reaches 0.032 and maintains it thereafter.

Except synchronization of two identical neurons, several different synchronized states of distinct neurons are also investigated. Similar results are obtained that all neurons will get synchronized when $g_{c}$ is larger enough as shown in Figure 6, in which we put all correlation coefficients with respect to the coupling strength $g_{c}$ together. But it can be observed from the local enlargement of the figure in the right corner that there are still some differences. In Figure 6, different states correspond to different line styles. It can be seen that CC of neurons $\left(V 1\left(T=3^{\circ} \mathrm{C}\right)\right.$ versus $\left.V 2\left(T=5^{\circ} \mathrm{C}\right)\right)$ and $\left(V 1\left(T=5^{\circ} \mathrm{C}\right)\right.$ versus $\left.V 2\left(T=7^{\circ} \mathrm{C}\right)\right)$ gets synchronized rapidly after being coupled with $g_{c}$, while these two states are all synchronized in single-spike pattern, which means that this state is more easy to be synchronized. CC of neurons $\left(V 1\left(T=32^{\circ} \mathrm{C}\right)\right.$ versus $\left.V 2\left(T=35^{\circ} \mathrm{C}\right)\right)$ is also increased rapidly, but slower than the above two cases because of their high frequencies. The most slowly synchronized neurons are $\left(V 1\left(T=5^{\circ} \mathrm{C}\right)\right.$ versus $\left.V 2\left(T=10^{\circ} \mathrm{C}\right)\right)$, in which two neurons are all in chaos states.

4.2. Synchronization of Neuronal Network. First, synchronization effects in neural networks coupled in ring-like manner are investigated. The network is connected by 25 coupled neurons as ring-like manner (Figure 3(b)). An ISIdistance method for multiple spike trains is used to give the quantitative results for synchronized spike trains [35]. First, the ISI for each spike train is assigned and by computing over all absolute $I S I$ ratios $\left|I_{m, n}(t)\right|$, the instantaneous average $A(t)$ is obtained as follows:

$$
\begin{aligned}
A(t) & =\frac{1}{N(N-1) / 2} \sum_{n=1}^{N} \sum_{m=n+1}^{N}\left|I_{m, n}(t)\right|, \\
D_{I}^{a} & =\frac{1}{T} \int_{t=0}^{T} A(t) d t .
\end{aligned}
$$

Average over time, $D_{I}^{a}$, is achieved. It is obvious that the more synchronous the multiple spike trains are, the smaller the ISI distance $D_{I}^{a}$ is; when complete synchronization state of the neuronal network is achieved, $D_{I}^{a}$ is equal to 0 . Then the effect of two parameters on synchronization of ringlike network is investigated. The contour graph of the ISI distance in the $\left(g_{c}, T\right)$ parameter plane is shown in Figure 7. 


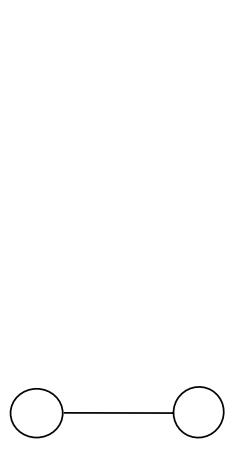

(a)

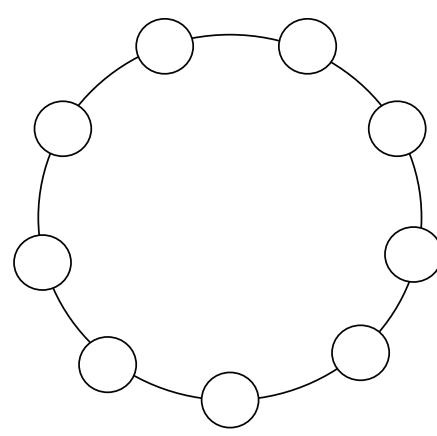

(b)

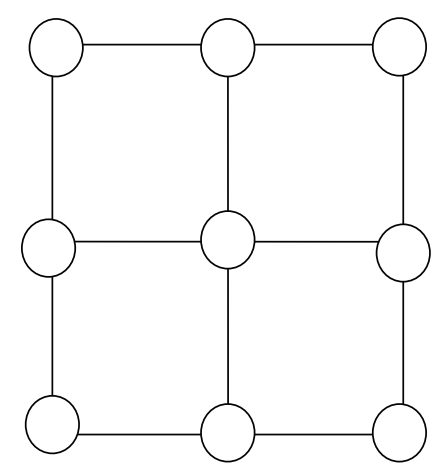

(c)

FIGURE 3: Structure of network connectivity patterns. (a) Two bidirectional coupled neurons. (b) A coupled ring-like neuronal network. (c) A coupled grid-like neuronal network.

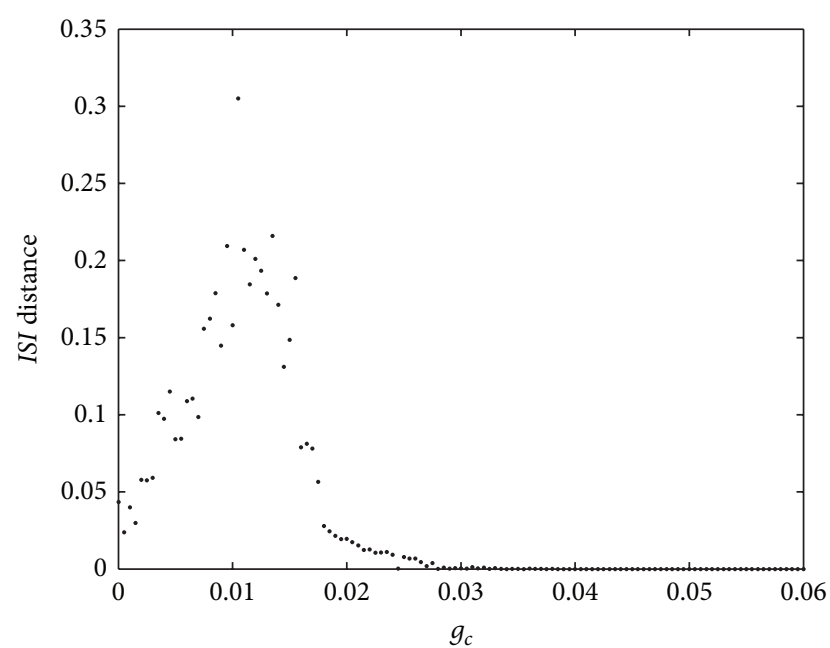

Figure 4: Plot of the ISI distance of two coupled neurons as a function of coupling strength $g_{c}$.

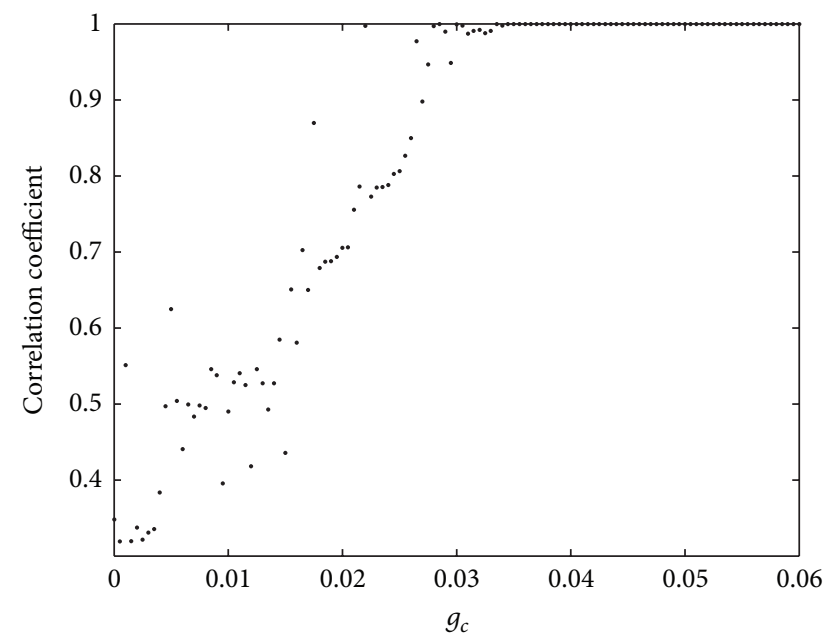

FIGURE 5: Plot of correlation coefficient of two coupled neurons as a function of coupling strength $g_{c}$.

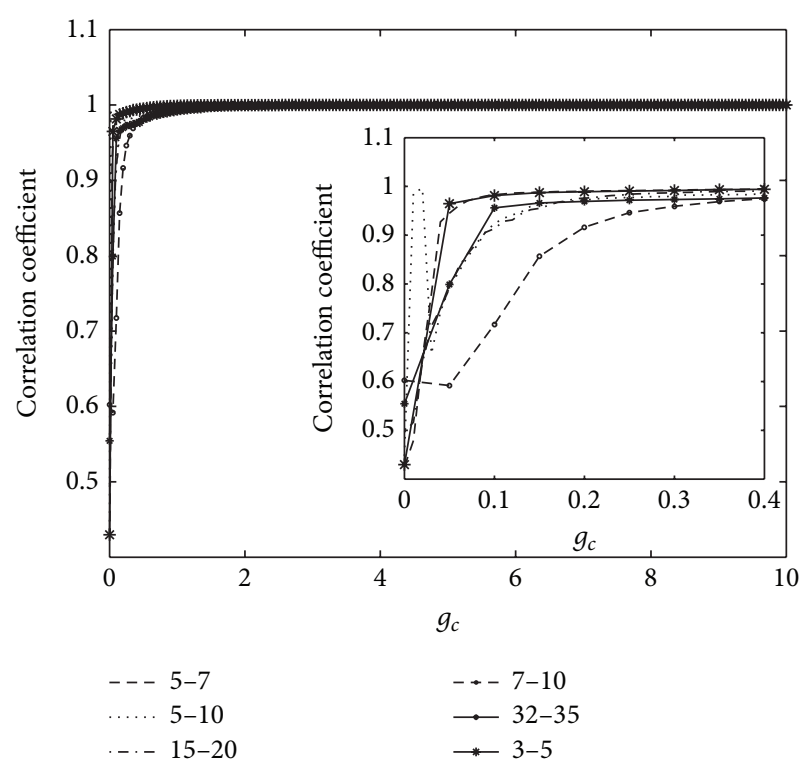

FIgURE 6: Correlation coefficient with respect to the coupling strength $g_{c}$ of all the states.

The plot shows that when coupling strength is bigger than $0.02 \mathrm{~ms} / \mathrm{cm}^{2}$, no matter what the value of $T$ is, the neurons are in a complete synchronous state. Also the region that ISI distance almost equals 0 is much larger than that of only changing one parameter $g_{c}$ shown as Figure 4. It indicates that the realization of synchronization in the ring-like neuron network is easier than that of two coupled neurons with changing only one parameter. Another obvious conclusion is that, no matter what the temperature $T$ is, when increasing the coupling strength, the neurons in the ring-like network can finally reach synchronization.

Next, to understand the relationship between synchronization and the network connection patterns, network connection type is changed to grid network. The number of neurons in the grid-like network is still 25. The coupling current of a neuron is the sum of the currents for the nearest neighbor neurons $(M=N=3)$. The corresponding contour graph 


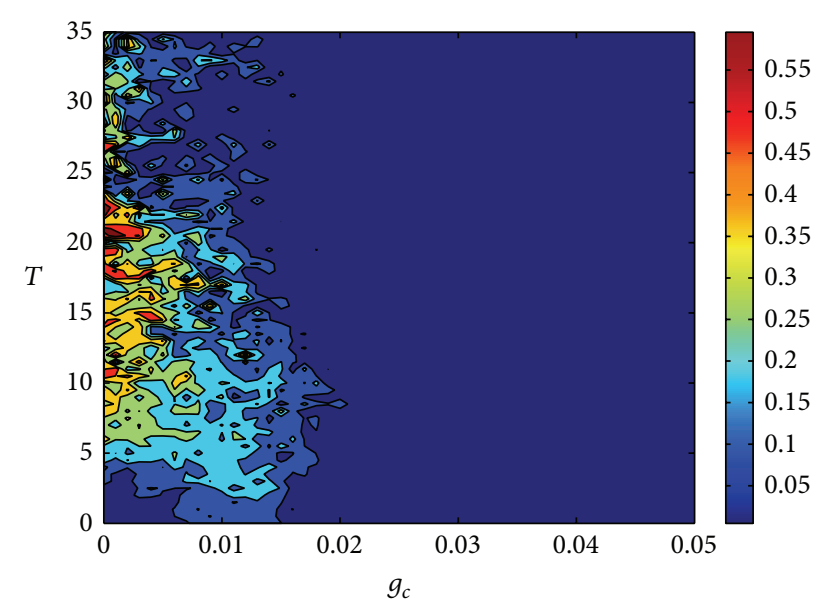

FIGURE 7: The contour plot of the ISI distance in the $\left(g_{c}, T\right)$ plane for ring-like network.

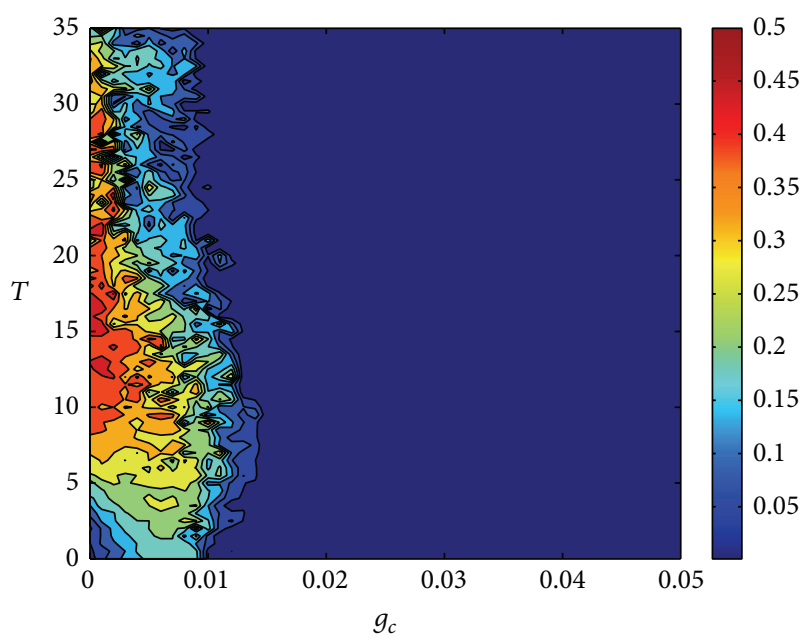

FIGURE 8: The contour plot of the average ISI distance in the $\left(g_{c}, T\right)$ plane for grid network.

of the ISI distance in the $\left(g_{c}, T\right)$ parameter plane is shown in Figure 8. As illustrated in the figure, the map differs from that of the ring-like neuronal network. It can be observed that the region that ISI distance almost equals 0 is larger than that of the ring-like neuronal network; when coupling strength is bigger than $0.012 \mathrm{~ms} / \mathrm{cm}^{2}$, all neurons are in a complete synchronous state. It indicates that synchronization state is related to the network connection type; that is, the synchronization in coupled grid-like neuronal network is easier than ring-like connected neurons. Also we can see from the simulation that, no matter what the $T$ is (the uncoupled neurons are in all kinds of firing patterns including regular single spike, chaotic, or bursting status), when the coupling strength is bigger enough, the grid-like neuronal network can eventually reach the complete synchronization, which is similar to ring-like network.

Compared to Figure 1, it can be observed from Figures 7 and 8 that $g_{c}$ of chaotic and high period pattern are larger than the ones in spike and low period patterns, which means that chaotic and high period patterns are more difficult to get complete synchronization than the situation in single spike and low period patterns. It can be also seen from these two figures that the tendency of ISI with temperature $T\left(g_{c}<0.02\right.$ in Figure 7 and $g_{c}<0.015$ in Figure 8) is consistent with the situation of single neuron, that is, increasing first and then decreasing. This means that it is possible to establish the relation between network dynamics and single-neuron dynamics.

\section{Conclusion}

First, the noise effects on the neuronal system are studied. With the addition of noise, the neural model can generate a variety of new different patterns compared to deterministic situation. For a qualitative computing of noise effect, the ISI distance is introduced to characterize how noise affects the variability of the neural spike train. It is shown that the ISI distance will increase with the noise intensity becoming larger, which means that the higher noise intensity can cause stronger fluctuations in the spike train. Meanwhile, noise has less effect on spike trains for higher temperature within the bursting regime. These stochastic phenomena mean that signal encoding can be different with the change of noise level.

Then synchronization status of neural network with different connection types is investigated. The simulations show that the synchronization status is much related to the network connectivity patterns. By tuning both temperature and coupling strength, there exists a larger synchronous region for grid-like neuronal network than that of ringlike network. It is also shown that chaotic and high period patterns are more difficult to get complete synchronization than the situation in single spike and low period pattern.

The method and results in this study may be instructive for the study of neural encoding of spike trains under noisy and network environment. Network study results show that it may be possible to establish the relation between network dynamics and single-neuron dynamics.

\section{Conflict of Interests}

The authors declare that there is no conflict of interests regarding the publication of this paper.

\section{Acknowledgments}

This work was supported by the National Natural Science Foundation of China (Grant nos. 11002055, 11232005, and U1204106) and the Special Fund of Civil Aviation University of China nos. 2012QD09X and ZXH2012C004.

\section{References}

[1] W. Horsthemke and R. Lefever, Noise-Induced Transitions: Theory and Applications in Physics, Chemistry and Biology, vol. 15 of Springer Series in Synergetics, Springer, Berlin, Germany, 1984.

[2] A. L. Hodgkin and A. F. Huxley, "A quantitative description of membrane current and its application to conduction and 
excitation in nerve," The Journal of Physiology, vol. 117, no. 4, pp. 500-544, 1952.

[3] P. Channell, G. Cymbalyuk, and A. Shilnikov, "Origin of bursting through homoclinic spike adding in a neuron model," Physical Review Letters, vol. 98, no. 13, Article ID 134101, 2007.

[4] D. Linaro, A. Champneys, M. Desroches, and M. Storace, "Codimension-two homoclinic bifurcations underlying spike adding in the Hindmarsh-Rose Burster," SIAM Journal on Applied Dynamical Systems, vol. 11, no. 3, pp. 939-962, 2012.

[5] R. Benzi, A. Sutera, and A. Vulpiani, "The mechanism of stochastic resonance," Journal of Physics A, vol. 14, no. 11, pp. L453L457, 1981.

[6] W. J. Freeman, "Deep analysis of perception through dynamic structures that emerge in cortical activity from self-regulated noise," Cognitive Neurodynamics, vol. 3, no. 1, pp. 105-116, 2009.

[7] B. Lindner, J. García-Ojalvo, A. Neiman, and L. SchimanskyGeier, "Effects of noise in excitable systems," Physics Reports, vol. 392, no. 6, pp. 321-424, 2004.

[8] M. Yang, Z. Liu, L. Li et al., "Identifying distinct stochastic dynamics from chao: a study on multimodal neural firing patterns," International Journal of Bifurcation and Chaos, vol. 19, no. 2, pp. 453-485, 2009.

[9] J. Mo, Y.-Y. Li, C.-L. Wei et al., "Interpreting a period-adding bifurcation scenario in neural bursting patterns using bordercollision bifurcation in a discontinuous map of a slow control variable," Chinese Physics B, vol. 19, no. 8, Article ID 080513, 2010.

[10] H. Gu, M. Yang, L. Li, Z. Liu, and W. Ren, "Dynamics of autonomous stochastic resonance in neural period adding bifurcation scenarios," Physics Letters A, vol. 319, no. 1-2, pp. 8996, 2003.

[11] Q. Lu, Z. Yang, L. Duan, H. Gu, and W. Ren, "Dynamics and transitions of firing patterns in deterministic and stochastic neuronal systems," Chaos, Solitons \& Fractals, vol. 40, no. 2, pp. 577-597, 2009.

[12] Q. Lu, H. Gu, Z. Yang, X. Shi, L. Duan, and Y. Zheng, "Dynamics of firing patterns, synchronization and resonances in neuronal electrical activities: experiments and analysis," Acta Mechanica Sinica, vol. 24, no. 6, pp. 593-628, 2008.

[13] H. A. Braun, M. T. Huber, M. Dewald, K. Schäfer, and K. Voigt, "Computer simulations of neuronal signal transduction: the role of nonlinear dynamics and noise," International Journal of Bifurcation and Chaos, vol. 8, no. 5, pp. 881-889, 1998.

[14] H. A. Braun, K. Schafer, K. Voigt et al., "Low-dimensional dynamics in sensory biology 1 : thermally sensitive electroreceptors of the catfish," Journal of Computational Neuroscience, vol. 4, no. 4, pp. 335-347, 1997.

[15] H. A. Braun, M. T. Huber, N. Anthes et al., "Noise-induced impulse pattern modifications at different dynamical periodone situations in a computer model of temperature encoding," BioSystems, vol. 62, no. 1-3, pp. 99-112, 2001.

[16] H. A. Braun, K. Voigt, and M. T. Huber, "Oscillations, resonances and noise: basis of flexible neuronal pattern generation," BioSystems, vol. 71, no. 1-2, pp. 39-50, 2003.

[17] H.-G. Gu, B. Jia, and G.-R. Chen, "Experimental evidence of a chaotic region in a neural pacemaker," Physics Letters A, vol. 377, no. 9, pp. 718-720, 2013.

[18] H. Gu, "Experimental observation of transition from chaotic bursting to chaotic spiking in a neural pacemaker," Chaos, vol. 23, no. 2, Article ID 023126, 2013.
[19] H. Gu, W. Ren, Q. Lu, S. Wu, M. Yang, and W. Chen, "Integer multiple spiking in neuronal pacemakers without external periodic stimulation," Physics Letters A, vol. 285, no. 1-2, pp. 6368, 2001.

[20] H. Gu, B. Jia, and Q. Lu, "Exponential decay characteristics of the stochastic integer multiple neural firing patterns," Cognitive Neurodynamics, vol. 5, no. 1, pp. 87-101, 2011.

[21] A. Pikovsky, M. Rosenblum, and J. Kurths, Synchronization: A Universal Concept in Nonlinear Scicencs, Cambridge Nonlinear Science Series, Cambridge Unversity Ress, New York, NY, USA, 2001.

[22] A. P. Davison, J. Feng, and D. Brown, "Spike synchronization in a biophysically-detailed model of the olfactory bulb," Neurocomputing, vol. 38-40, pp. 515-521, 2001.

[23] W. Singer, Time as Coding Space in Neocortical Processing, Springer, Berlin, Germany, 1994.

[24] R. Eckhorn, "Neural mechanisms of scene segmentation: recordings from the visual cortex suggest basic circuits for linking field models," IEEE Transactions on Neural Networks, vol. 10, no. 3, pp. 464-479, 1999.

[25] S. Postnova, K. Voigt, and H. A. Braun, "Neural synchronization at tonic-to-bursting transitions," Journal of Biological Physics, vol. 33, no. 2, pp. 129-143, 2007.

[26] M. Nomura, T. Fukai, and T. Aoyagi, "Synchrony of fastspiking interneurons interconnected by GABAergic and electrical synapses," Neural Computation, vol. 15, no. 9, pp. 2179-2198, 2003.

[27] W. J. Freeman, Neurodynamics: An Exploration in Mesoscopic Brain Dynamics, Springer, London, UK, 2000.

[28] S. Postnova, C. Finke, W. Jin, H. Schneider, and H. A. Braun, "A computational study of the interdependencies between neuronal impulse pattern, noise effects and synchronization," Journal of Physiology, vol. 104, no. 3-4, pp. 176-189, 2010.

[29] Y. D. Sato and M. Shiino, "Generalization of coupled spiking models and effects of the width of an action potential on synchronization phenomena," Physical Review E, vol. 75, no. 1, Article ID 011909, 15 pages, 2007.

[30] Q. Y. Wang, Q. S. Lu, and Z. S. Duan, "Adaptive lag synchronization in coupled chaotic systems with unidirectional delay feedback," International Journal of Non-Linear Mechanics, vol. 45, no. 6, pp. 640-646, 2010.

[31] W. Yu, J. Cao, and W. Lu, "Synchronization control of switched linearly coupled neural networks with delay," Neurocomputing, vol. 73, no. 4-6, pp. 858-866, 2010.

[32] Y.-Q. Che, J. Wang, K.-M. Tsang, and W.-L. Chan, "Unidirectional synchronization for Hindmarsh-Rose neurons via robust adaptive sliding mode control," Nonlinear Analysis: Real World Applications, vol. 11, no. 2, pp. 1096-1104, 2010.

[33] Y. Hao, Y. Gong, X. Lin, Y. Xie, and X. Ma, “Transition and enhancement of synchronization by time delays in stochastic Hodgkin-Huxley neuron networks," Neurocomputing, vol. 73, no. 16-18, pp. 2998-3004, 2010.

[34] J. Ma, F. Li, L. Huang, and W.-Y. Jin, “Complete synchronization, phase synchronization and parameters estimation in a realistic chaotic system," Communications in Nonlinear Science and Numerical Simulation, vol. 16, no. 9, pp. 3770-3785, 2011.

[35] T. Kreuz, D. Chicharro, R. G. Andrzejak, J. S. Haas, and H. D. I. Abarbanel, "Measuring multiple spike train synchrony," Journal of Neuroscience Methods, vol. 183, no. 2, pp. 287-299, 2009. 
[36] T. Kreuz, J. S. Haas, A. Morelli, H. D. I. Abarbanel, and A. Politi, "Measuring spike train synchrony," Journal of Neuroscience Methods, vol. 165, no. 1, pp. 151-161, 2007.

[37] R. F. Fox, I. R. Gatland, R. Roy, and G. Vemuri, "Fast, accurate algorithm for numerical simulation of exponentially correlated colored noise," Physical Review A, vol. 38, no. 11, pp. 5938-5940, 1988. 


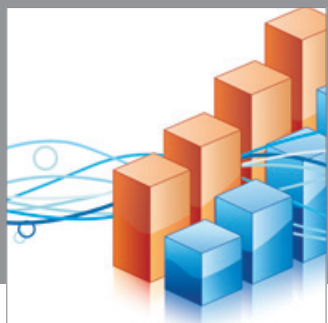

Advances in

Operations Research

mansans

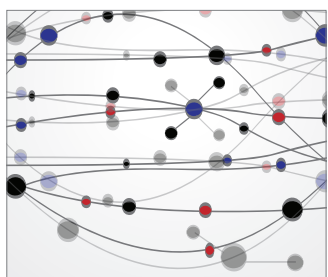

The Scientific World Journal
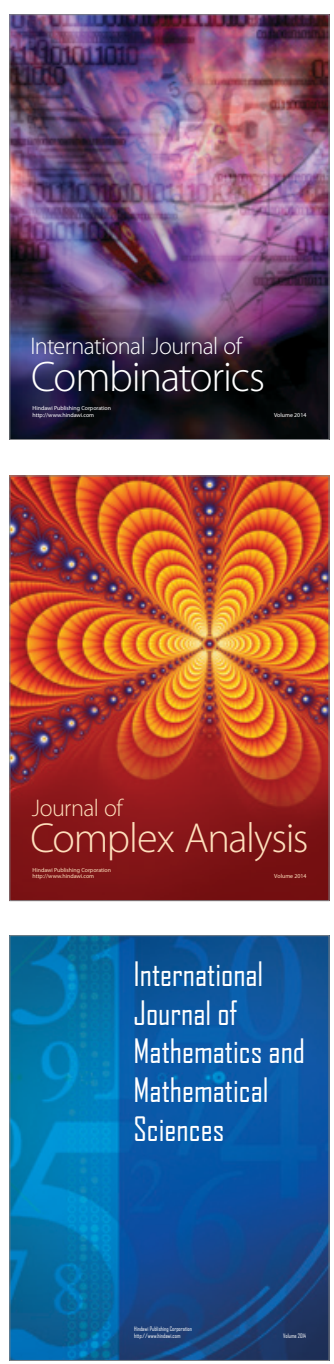
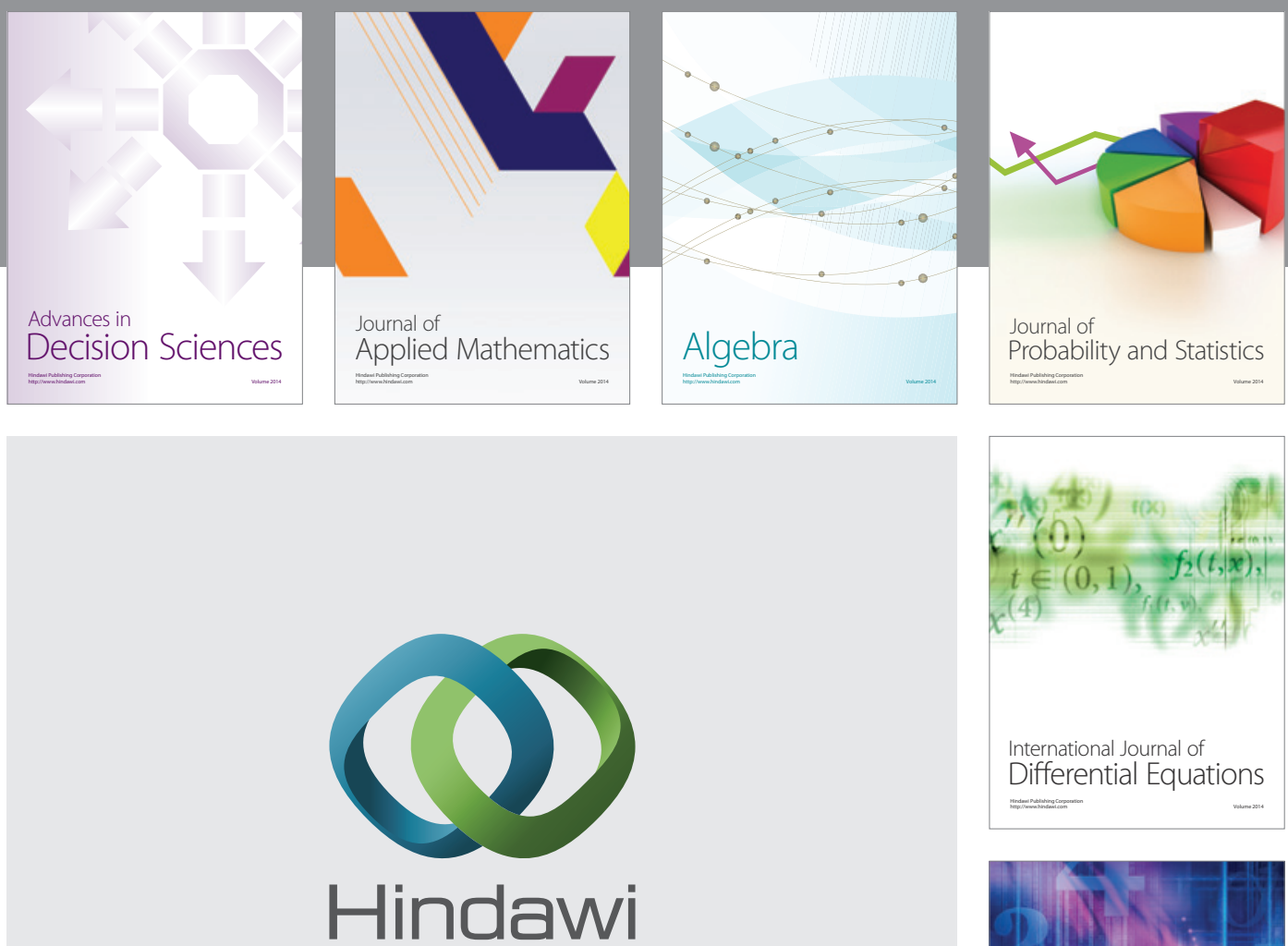

Submit your manuscripts at http://www.hindawi.com
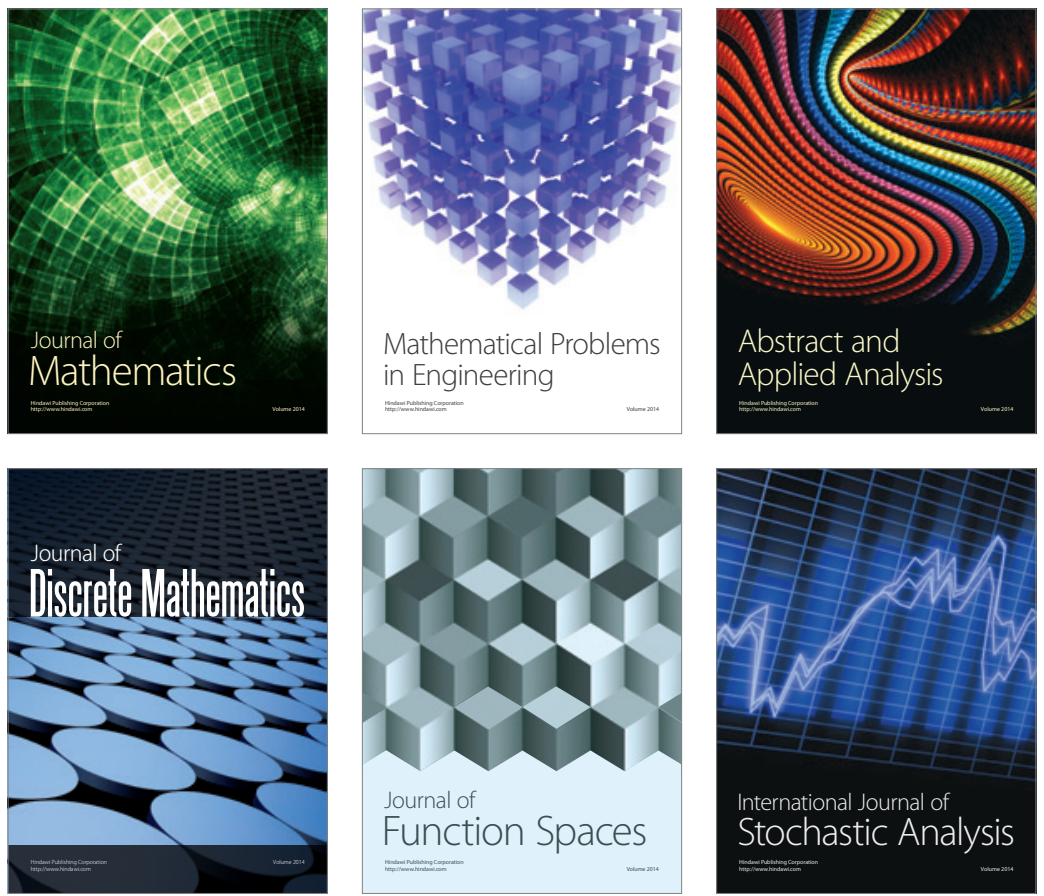

Journal of

Function Spaces

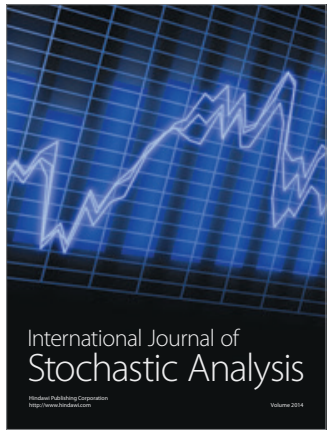

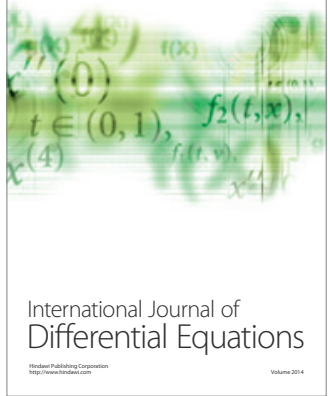
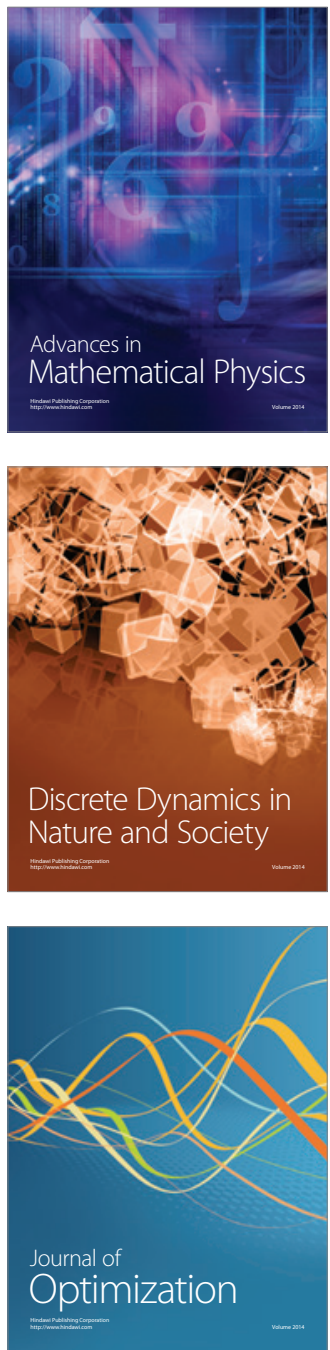